Sociologie et sociétés

\title{
Table ronde : le Département de sociologie de l’Université de Montréal
}

\section{Forum - The Department of Sociology of the University of Montreal}

\author{
Jacques DOFNY, Hubert GUINDON, Norbert LACOSTE et Marcel RIOUX
}

Volume 12, numéro 2, octobre 1980

Réflexions sur la sociologie

URI : https://id.erudit.org/iderudit/001189ar

DOI : https://doi.org/10.7202/001189ar

Aller au sommaire du numéro

\section{Éditeur(s)}

Les Presses de l'Université de Montréal

\section{ISSN}

0038-030X (imprimé)

1492-1375 (numérique)

Découvrir la revue

Citer ce document

DOFNY, J., GUINDON, H., LACOSTE, N. \& RIOUX, M. (1980). Table ronde : le Département de sociologie de l'Université de Montréal. Sociologie et sociétés, 12(2), 179-201. https://doi.org/10.7202/001189ar

\section{Résumé de l'article}

Publié à l'occasion du vingt-cinquième anniversaire du Département de sociologie de l'Université de Montréal, ce numéro porte sur l'histoire et le développement de ce Département. Ce document prend la forme d'une table ronde à laquelle ont participé Jacques Dofny, Hubert Guin-don, Norbert Lacoste et Marcel Rioux. On y trouvera des références aux divers courants sociologiques qui influencèrent (ou non) les premières années de ce Département, aux positions prises par divers acteurs de l'institution universitaire, au contexte social et culturel du Québec de la fin des années cinquante, aux mouvements étudiants, etc. Ce document présente à la fois des éléments d'analyse et des témoignages personnels de personnes qui ont toutes été intimememt liées au Département de sociologie dans les premières années de son existence. Cette table ronde était animée par Robert Sévigny. 


\section{DOCUMENT}

\section{Table ronde \\ Le Département de sociologie de l'Université de Montréal}

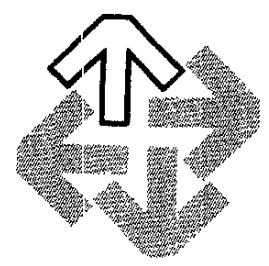

Participants :

JACQUES DOFNY, HUBERT GUINDON, NORBERT LACOSTE et MARCEL RIOUX

Animateur :

ROBERT SÉVIGNY

Dans ce numéro publié à l'occasion du vingt-cinquième anniversaire du Département de sociologie de l'Université de Montréal, nous avons pensé inclure un document sur l'histoire et le développement de ce Département. $\mathrm{Ce}$ document prend la forme d'une table ronde à laquelle ont participé Jacques Dofny, Hubert Guindon, Norbert Lacoste et Marcel Rioux. On y trouvera des références aux divers courants sociologiques qui influencèrent (ou non) les prernières années de ce Département, aux positions prises par divers acteurs de l'institution universitaire, au contexte social et culturel du Québec de la fin des années cinquante, aux mouvements étudiants, etc. Ce document présente à la fois des éléments d'analyse et des témoignages personnels de personnes qui ont toutes été intimememt liées au Département de sociologie dans les premières années de son existence. Cette table ronde était animée par Robert Sévigny.

R.S. - Cette table ronde sera publiée à l'intérieur d'un numéro de Sociologie et sociétés publié à l'occasion du $25^{\mathrm{e}}$ anniversaire du Département. Le titre de ce numéro sera «Réflexions sur la sociologie». Ceci veut donc dire qu'il n'y aura pas seulement des textes sur le Département de sociologie, mais aussi des réflexions sur les courants de la sociologie, sur la sociologie et les disciplines connexes et sur les champs d'appli- 
cation de la sociologie, etc. Certains thèmes que l'on va aborder entre nous vont aller dans le sens de textes qui vont être publiés, mais l'idée principale de cette table ronde, c'est de profiter de la présence de quatre personnes qui ont connu le Département assez bien à différents moments dans le passé, pour essayer de réfléchir tout haut sur des questions comme : quels sont les types de sociologies qui y ont été enseignées et qui s'y enseignent? Quelles sont les caractéristiques des étudiants? Quelles sont les fonctions de la sociologie ou du Département à l'égard de la société, soit du point de vue des professeurs, soit du point de vue des étudiants qu'on prépare. À quels métiers de sociologue on prépare les étudiants ?, etc. Tout ça, par ailleurs, pourrait être discuté en tenant compte, en arrière plan, du développement du Québec durant cette période, du développement de la sociologie elle-même, et de bien d'autres variables : chacun pourra évidemment faire référence au cadre d'analyse qui l'inspire dans ses réflexions sur le développement du Département depuis ces vingt-cinq ans. Une façon d'amorcer nos échanges serait d'indiquer quelles sont quelques dates importantes qui donnent un sens à l'histoire du développement du Département. Je ne sais pas si, à partir de là, on peut demander à Norbert Lacoste, qui est parmi nous le doyen de lancer la première balle et de nous dire qu'elles sont pour lui quelques-unes de ces dates importantes.

N.L. - La première date qui me semble importante est vraiment dans la préhistoire du Département de sociologie : c'est 1948. En 1948, Monseigneur Joseph Charbonneau, alors archevêque de Montréal et chancelier de l'Université me dit: «Nous allons avoir de grands changements au Québec d'ici quelques années, il faut vous préparer à comprendre ce qui va se passer.» Et c'est ainsi qu'il m'envoie étudier les sciences sociales à Louvain. Je reviens en 1951 et on me demande de donner le premier cours de sociologie. Ça se donnait au cours du soir. J'avais, parmi mes élèves brillants, Alfred Dubuc. C'était dans le cadre de la faculté des sciences sociales dont le doyen était Esdras Minville. On a donné des cours jusqu'en 55, et à ce moment-là, Mgr Lussier, qui était le recteur de l'Université, m'a demandé de diriger le nouveau Département de sociologie, fondé le 15 mars 1955. En même temps, à la même date, était fondé le Département de sciences économiques qui était dirigé par André Raynauld. Il y avait déjà à ce moment-là, deux autres Départements qui existaient : celui des relations industrielles qui était dirigé par L. P. Brizard, et le Père Guillemette o.p. dirigeait l'École de service social. C'est vraiment 1955 , je pense, qui est l'année marquante au Département. Pour les autres dates importantes, je pense que lorsque le Département est devenu autonome par rapport à la faculté des sciences sociales, cela a sûrement été une autre date importante. Je parlerai des préoccupations que nous avons eues dans le recrutement et les perspectives que nous avions à ce moment-là.

R.S. - On peut tout de suite aborder le développement de cette période-là, si vous voulez.

N.L. - Ce qui a commandé le développement à ce moment-là, c'était vraiment le rattrapage. L'Université Laval avait déjà un très bon Département 
de sociologie. Nous avions mauvaise réputation par rapport à Laval et notre premier objectif était vraiment compétitif par rapport à celle-ci. Nous avons immédiatement essayé de trouver et d'attirer au Département des sociologues qui auraient pu contribuer à son développement. C'est pourquoi au lieu de nous enfermer dans une perspective assez unique et de faire des conflits de chapelle, on a essayé immédiatement de s'adjoindre de jeunes sociologues qui avaient une formation, soit européenne, soit américaine. C'est ainsi que Hubert Guindon était de ceux que l'on avait recrutés à ce moment-là : il y avait eu également Paule Verdet, Sally Cassidy, Fernand Cadieux. C'était le tout premier noyau avant 1955 qui commençait à se réunir. À ce moment, on essayait d' «attraper», je dirais, tout ce qui pouvait être intéressant autour, mais notre modèle était le programme de Laval. On essayait de mettre sur pied une institution qui était comparable à ce modèle-là. Par la suite évidemment, on s'est distingué de Laval avec une trajectoire plus originale à mon point de vue.

R.S. - Mais si on s'en tient à cette période-là, comment caractériseriez-vous maintenant les étudiants que vous aviez alors.

N.L. - Les étudiants étaient des gens souvent orientés vers les sciences sociales, mais à qui il manquait généralement une formation de base au point de vue théorique. Les premiers cours étaient vraiment pour donner à ces étudiants-là, des assises plus fondamentales sur le fonctionnement de la société. Les premières thèses que l'on a dirigées ont été celle de Pierre Dandurand, dont le thème était «l'étude de contenu des éditoriaux du Devoir depuis 1910; il y avait aussi Marcel Dagenais qui est devenu économiste, qui avait étudié le mécanisme de la conversion dans l'Inquiry forum qui existait à ce moment-là ; il y avait aussi une étude qui avait été faite sur une secte religieuse dans la ville de TroisRivières par Jean-Marie Rainville; il y avait Marie-Pia Joanette qui a fait une thèse sur la sélection des élèves et la promotion sociale dans la ville de Sainte-Agathe. Eliette Leblanc et le turn-over des gardesmalades à l'hôpital Notre-Dame. C'était la période des monographies. Nous avons formé, à ce moment, des étudiants qui reçurent une formation empirique. On a essayé de se spécialiser un peu dans différentes matières. Il y avait l'organisation sociale et la psychologie sociale; il y avait déjà les structuralistes de l'époque. On a recruté un certain nombre de collègues dont le centre d'intérêt était particulier, qui, très tôt, ont donné naissance à d'autres départements. On est allé chercher Szabo à Lyon, qui est devenu responsable de la criminologie ; Garigue à l'Université McGill qui est ensuite passé au Département de science politique après être devenu doyen; Brazeau à Toronto qui avait eu une expérience à Chicago; Dubreuil et Henripin, ont donné naissance aux Départements d'anthropologie et de démographie.

M. R. - Donc il y avait la filière Louvain, la filière américaine et un petit peu la filière française.

N. L. - C'est ça. À ce moment-là (1960), Chicago était représenté par Brazeau. 
M.R. - En fait il y avait Henripin qui avait fait ses études à Paris. Donc, dès le départ, le Département de sociologie est assez pluraliste, très diversifié. Mais à ce moment-là est-ce qu'il fallait être catholique pour accéder au Département?

N.L. - Non ! À ce moment-là ce que l'on demandait c'était une tolérance et un respect des institutions en place mais il n'y avait pas de critères religieux personnels exigés des professeurs.

R.S. - Hubert, toi, tu as enseigné ici durant cette période, jusqu'en 1959$1960 \ldots$

H.G. - Moi, à l'encontre de l'abbé Lacoste, je n'ai jamais eu le privilège, même jusqu'à aujourd'hui, de parler aux évêques et aux archevêques, mais l'image que j'ai du début du Département c'est un peu celle-là. L'idée de copier Laval ne m'avait pas semblé aussi forte, mais c'était plutôt l'abbé Lacoste qui était en charge des programmes. La tendance générale, c'est que les jeunes professeurs prennent toujours un peu copie carbonne ou un modèle de l'institution d'où ils sortent. Chicago donc avait une influence assez forte parce que Sally Cassidy comme Paule Verdet et moi-même, venions tous les trois de Chicago, même si je ne les avais pas connus à l'Université de Chicago.

J. D. - À cette période-là l'influence était la sociologie américaine...

H.G. - C'était une influence, probablement dans les trois cas, assez prépondérante de George Mead de la psychologie sociale de l'école de Chicago. Ce qui fait qu'il y avait un intérêt assez marqué pour ce qu'on pourrait appeler quasiment de la sociologie anthropologique, c'est-à-dire un peu, du travail sur le terrain, tout l'aspect de la signification des conduites. Je pense que parallèlement aussi la collaboration de Fernand Cadieux avec la fondation du Groupe de recherche sociale a marqué l'orientation des premiers étudiants. C'était un gradué de Laval en économique, mais il avait quand même une perspective assez large des sciences sociales.

M.R. - ...Américanophile!

H.G. - Américanophile, mais remarquez qu'être francophile en 1955 !... il n'y avait pas grand-chose en France ; c'était l'éclosion peut-être de l'existentialisme mais au niveau de la sociologie c'était une période de reconstruction, d'après-guerre... Du moins c'était la perception qu'on avait. Mais en réalité, on ne regardait pas de ce côté-là.

J.D. - Sur l'affaire de Cadieux, tu pourrais développer un peu? Qu'est-ce que c'était?

H.G. - Ce Groupe de recherche sociale, d'après moi, se voulait plus ou moins copié sur le Bureau of Applied Social Research de l'Université Columbia. Ça a regroupé du monde qui au début sortait de la filière de la J.E.C.

J.D. - Qui entre autres?

H.G. - Il y avait les deux frères Breton, Raymond et Albert, il y avait Fernand Cadieux. C'était vraiment je pense Fernand Cadieux qui était au centre 
de ce projet-là. Les deux autres c'était les deux collaborateurs, l'un en économique, l'autre en sociologie.

M.R. - Pinard, Maurice Pinard?

H.G. - Maurice Pinard quand il est revenu d'Europe. Il était sorti du droit, il était notaire. D'ailleurs il avait gagné le prix des notaires de la province et il a laissé le notariat en s'en allant en sociologie en France, d'où il est revenu quelque temps plus tard. Là on arrive vers la fin des années 50. À ce moment, ce monde-là s'est redirigé vers Chicago ou vers Johns Hopkins. Il n'y avait pas de lien organique entre le G.R.S. et l'Université de Montréal, mais il y en avait du fait de la nature des travaux. Il y avait pas mal d'étudiants qui indépendamment de leur programme de sociologie, étaient embauchés au niveau de la recherche, prenaient probablement une certaine expérience de travaux empiriques et de travail en groupe, par équipe. Là on pourrait mentionner des personnes comme Rodrigue Johnson, Jean-Pierre Alix, même Pierre Dandurand, je pense, y a travaillé; Jacqueline Massé peut-être pas directement mais elle était un peu dans cette orbite-là. Elle est allée étudier directement à Chicago...

R.S. - Quels furent les grands débats à ce moment-là?

H.G. - Les débats au niveau de l'idéologie, je ne sais pas si vous allez être d'accord ou pas. Je pense que Guy Rocher a écrit un texte qui à mon avis est assez sensible à la tonalité émotive de l'époque. Il le donnait à Winnipeg il y a plusieurs années. Il vaudrait la peine de le retrouver. C'était Minville qui était doyen et à ce moment-là, les autorités avaient une définition technique de la sociologie; ils voyaient la sociologie un peu comme une technique de ramassage de données empiriques. Le problème de l'interprétation des données par exemple, ça c'était implicitement la compétence des autorités alors que nous les sociologues, à ce moment-là, voulions avoir notre indépendance, pas seulement pour ramasser les données, mais pour les interpréter nous-mêmes également. Il y avait une tension là-dedans mais qui n'était pas vive. Elle n'était pas vive parce qu'on ne faisait pas tellement de travaux empiriques: on était plutôt engagés dans l'enseignement.

N.L. - On avait fait tout de même une petite contestation vis-à-vis du doyen parce que, à ce moment-là, Esdras Minville était très nationaliste et il avait voulu orienter la recherche dans un sens particulier. Hubert et moi nous avions contesté.

H.G. - Oui, au niveau des grands débats de l'époque, ça se situait entre deux pôles. Il y a eu le Fédéralisme canadien de Lamontagne, en 56 je crois. Le thème du fédéralisme était un des grands débats des sciences sociales ailleurs, mais il n'impliquait pas tellement les sciences sociales de Montréal; c'était plutôt l'école d'histoire (à l'Université de Montréal) et les sciences sociales de Laval. Au Département de sociologie, on était tous spectateurs de ça...

M.R. - C'est la question que j'allais vous poser parce que les années 50 sont marquées, en 1950 d'abord, par la fondation de Cité libre. À ce moment- 
là je suis au Département d'anthropologie du Musée de l'homme et j'embarque dans Cité libre. Mais ceux qui sont avec nous ce sont les sociologues de Laval et, à plusieurs reprises, on a des réunions avec des sociologues de Laval et des gens de Cité libre (Trudeau et les autres, n'est-ce pas) pour savoir ce que l'on fait. Comment se fait-il que vous n'êtes pas présents dans cette équipe ou dans ce débat? Parce que c'est l'époque de la contestation de la société traditionnelle et que l'abbé Lacoste, qui était directeur du Département de sociologie, ne voulait pas contester l'Église, les cultures traditionnelles? C'est la question que je vous pose.

R.S. - Je vous préviens que je vais vous demander votre propre réponse à votre question.

N.L. - À ce moment-là Laval regardait Montréal comme un parent pauvre. On s'était tourné vers les sociologues disponibles qui étaient plus empiriques.

H.G. - Moi j'avais une autre opinion. C'est que d'abord les trois personnes que vous avez mentionnées, c'est-à-dire Paule Verdet, Sally Cassidy et moi-même, on avait été absents aux études. Eux n'étaient pas Canadiens d'origine, donc c'était un conflit dans lequel ils se sentaient peu à l'aise, n'ayant pas vécu ici. Moi j'avais été quatre ans à Chicago et lorsqu'on est à l'étranger, les confiits du pays - peut-être pas aujourd'hui mais à l'époque - ce n'était pas les mass-communications comme aujourd'hui, alors on ne pouvait pas suivre aussi bien les conflits de son pays. L'autre raison c'est qu'on n'était pas invité, parce que je pense, que d'après ce que vous venez de dire, que les relations avec Laval n'étaient pas mauvaises mais elles étaient inexistantes. On n'avait pas émergé à l'horizon du ciel sociologique, du moins dans la pensée de ceux qui étaient à Laval.

M.R. - Je vous rappellerai que Cité libre était une fondation montréalaise, une revue montréalaise!

H. G. - Oui mais on n'était pas en contact avec Pierre Trudeau non plus.

M.R. - Mais Fernand...

H.G. - Fernand Cadieux était en contact...

M.R. - Comment ce fait-il que ça n'a pas transpercé dans votre milieu et que Cadieux lui-même, si je me souviens bien, n'a pas collaboré beaucoup, peu ou pas, pendant ces dix années 50-60?

H.G. - À Cité libre ou bien...?

M.R. - À Cité libre, qui était un mouvement intellectuel important. On a fait des thèses là-dessus. Je crois que ça a donné naissance à toutes sortes d'autres mouvements et cela, au niveau social...

N.L. - Je pense que l'on était, à ce moment-là, beaucoup plus empiriste. On recherchait surtout des monographies. Les théories globales devenues célèbres ne nous touchaient pas tellement. 
M.R. - Est-ce que ça veut dire que l'influence américaine prédominait à ce moment-là dans le Département?

N.L. - Oui, elle était sûrement importante.

H.G. - L'influence américaine c'est sûr... on ne la percevait pas comme une influence américaine... c'était l'influence de la sociologie! Maintenant l'autre aspect, c'est qu'on était seulement trois ou quatre personnes d'ailleurs ça fluctuait assez - et on enseignait 15-20 heures par semaine ce qui ne permettait pas tellement de participer à ces débats.

R.S. - (à M.R.) Est-ce que je peux vous demander votre réponse à votre question?

M.R. - Si vous demandez des dates privilégiés, je dirais 60 , 68 et 76 . Je pense que l'influence du Département de sociologie de l'Université de Montréal a été maximale durant ces huit premières années, de 1960 à 1968, semble-t-il. C'est l'époque où la sociologie au Département a été polyvalente et remplaçait plus ou moins le droit. Nous avions des cohortes d'étudiants extrêmement nombreuses et ces étudiants-là se sont ensuite dispersés dans la société, dans tous les azimuts. Par exemple au bac en 67 ou 68 , il y avait 275 étudiants en première année, sans compter les autres années. Alors à ce moment-là il me semble que l'influence du Département de sociologie a été prépondérante et il me semble qu'à partir de 68 c'est allé en diminuant. Ce que je veux dire dans le fond c'est que cette influence maximale sur la société existait parce que nous enseignions une sociologie qui était peut-être moins spécialisée et plus générale. Les sociologues, déjà à ce moment-là, faisaient partie, si vous voulez, de ces idéologues contestataires; nous étions engagés dans la société et en fondant des revues, et en participant à des partis politiques et en faisant ou en continuant de faire le procès de la société traditionnelle et des institutions traditionnelles.

H.G. - Ce que vous faites remarquer, c'est vrai. Dans toutes les universités, de 60 à 68, ce fut l'époque, en Amérique du Nord, de la grande croissance.

M.R. - Mais jusqu'à ce moment-là il n'y avait que deux seuls Départements de sociologie au Québec qui étaient Laval et Montréal et bien sûr que la naissance de l'UQAM a une influence sur le Département de sociologie. Déjà, nous sommes perçus comme une université bourgeoise, un département plus rangé par rapport à celui de l'UQAM qui veut devenir une université ouverte sur le monde avec beaucoup plus de participation. Je me souviens de quelqu'un qui parlait de «swinging university» et qui était un des fondateurs; et bien sûr que ça a influencé le public étudiant et leurs parents; le Département de sociologie, l'Université du Québec à Montréal devient beaucoup plus à l'image d'une université populaire que cette Université de Montréal, qui est déjà rangée, qui a des lettres de noblesse... ayant eu un abbé Lacoste à sa direction!

R.S. - À ce moment-ci, est-ce que vous décrivez la façon dont l'Université de Montréal et le Département de sociologie étaient perçus ou décrivezvous votre propre perception? 
M.R. - Moi je crois que j'ai tendance à vouloir marcher avec la société. Je pense que mon engagement, mes options théoriques ou idéologiques ont beaucoup à faire avec l'évolution du Québec et je distingue mal les deux.

N.L. - La première période a consisté à s'initier aux disciplines de la sociologie et la deuxième période à analyser le Québec. Le passage d'une définition idéologique à une définition scientifique, a constitué une période d'importance. Il faudrait également parler de l'influence marxiste au Département. Très tôt je me suis posé le problème : «Qu'est-ce qu'on fait vis-à-vis de cette vague très bien caractérisée dans l'Église et associée au mouvement communiste ? Que fait-on vis-à-vis de cette idéologie qui entre au Département?» Je me rappelle m'être posé la question et m'être dit: "C'est un mouvement qui va déferler; il faut l'accepter et être capable de surnager. " C'est ce qui s'est passé. Il y a eu une vague marxisante extrêmement puissante; l'élément valable de l'analyse sociale marxiste a été retenu tandis que les aspects idéologiques ont été étudiés par d'autres disciplines. Je pense que cela a contribué à donner au Département de sociologie deux approches : une approche wébérienne qui était dans la tradition chrétienne, et une approche marxienne, de tendance matérialiste. Je pense que cela a donné au Département la pluralité d'approche qui en fait sa richesse.

M.R. - Juste un mot sur ce qui précède 1960 . Je me souviens qu'à ce momentlà il avait été question de moi comme doyen de la faculté des sciences sociales. J'avais eu une entrevue avec monsieur Minville qui était à ce moment-là doyen; ce n'était pas tant que $\mathbf{M}$. Minville s'opposait au père Lévesque mais le père Lévesque s'était dit «on a la vérité mais allons confirmer ça dans les faits ». C'était là une forme d'empirisme, tandis que je crois que Minville n'est jamais sorti de l'idée que, possédant la vérité et marchant plutôt par déduction que par induction, il voyait assez mal ce que ça viendrait corroborer de ses convictions religieuses et nationales, et tout ce que vous voulez. Est-ce que votre position n'était pas un peu en porte-à-faux avec votre doyen? On voit que le Citoyen canadien-français que Minville publie en trois volumes à la fin de sa carrière, c'est une démarche déductive (le Québécois, le Français...). Il n'y a pas d'empirisme du tout, bien qu'il ait fait des recherches à Grande Vallée ou ailleurs. Ce qui l'intéresse, c'est une idéologie, une théorie de l'intégration de la recherche dans une vision catholique des choses. La recherche semblait un peu superflue...

N.L. - J'ai assisté à une rencontre entre le doyen qui était aussi à ce moment-là directeur des Hautes Études commerciales et le Père Eichmann un célèbre dominicain médiéviste: "Mon Père, disait M. Minville, maintenant que je suis doyen des sciences sociales, je voudrais reconstruire une société à partir des principes de l'Église.» Et le Père Eichmann de lui répondre: «Monsieur le doyen ou bien vous reconstruisez en fonction des principes du Moyen Âge et à ce moment-là c'est toute la société qu'il faut faire revenir au Moyen Âge, ou bien vous construisez une société pour le monde d'aujourd'hui et vous partez de la situation actuelle.» M. Minville était un homme d'une grande sincérité mais il 
avait un modèle de pensée déductive, et ne concevait pas comment on pouvait rebâtir une société de façon empirique. De là le conflit dont on parlait tout à l'heure entre M. Minville, Hubert Guindon et moi-même qui étions les jeunes du Département.

H. G. - Le conflit en partie est devenu plus aigu à l'occasion d'un geste politique de M. Minville à propos de l'acceptation ou de la non-acceptation des subsides du gouvernement fédéral aux universités. On était six à l'avoir désavoué publiquement dans un communiqué aux journaux. Je me souviens c'est vous (N.L.) qui aviez rédigé le communiqué. On se «désolidarisait» mais on avait employé le mot "désavoué» pour titrer la nouvelle, ce qui était techniquement correct, mais c'est une expression beaucoup plus forte par ici que la première... Il faut se souvenir qu'il n'y avait pas de budgets per capita. Notre position et celle du recteur étaient: «Peu importe d'où venait l'argent.»C'était devenu une pierre d'achoppement dans le développement des institutions universitaires, et M. Minville supportait la position du Premier ministre. Probablement le seul geste public à peu près qui s'est fait à l'intérieur du cadre de la faculté des sciences sociales, geste d'un homme assez important parmi les personnes de l'époque et geste un peu collectif. Par ailleurs, au niveau idéologique, je croirais moi que l'enjeu était d'arracher l'autonomie de la sociologie comme discipline; c'était la principale tension qu'il pouvait y avoir entre l'autorité et nous: l'autonomie de la discipline contre l'idée d'une sociologie définie techniquement. D'un autre côté, à l'intérieur du Département, il y avait une certaine tension entre ceux qui étaient de l'École de Chicago, qui étaient de tendance interactioniste et entre ceux du néopositivisme catholique. On pourrait définir que le néopositivisme, c'est anti-catholique par définition, autant que le marxisme! Mais le néopositivisme catholique, c'était une perception du rôle des sociologues comme étant des faiseurs de questionnaires, des ramasseurs d'entrevue et des codificateurs (à l'époque c'était la carte que l'on poinçonnait à la main...). Le néopositivisme catholique visait à un empirisme non dangereux et de service et, à mon avis, les tensions qui existaient à l'époque tournaient autour de cette question.

- Je peux vous conter des histoires. En 55 je pense, William F. Buckley écrit son volume qui a fait rage à l'époque God at Yale et qui déplorait l'athéisme dans les universités. Puis en 56 il y avait un étudiant qui a changé de McGill à l'Université de Montréal en économique, un nommé Hudon qui est mort dans un accident de la route plusieurs années plus tard. Je me souviens du cours de sociologie de l'époque... Hudon avait demandé qu'on commence tous les cours avec la prière au SaintEsprit. Il y en a qui la disaient, il y en a qui ne la disaient pas, la plupart des laïcs ne la disaient pas (moi parce que je ne m'en souvenais pas!) mais alors quand il l'avait demandé ça avait fait parler. Les professeurs d'économique, Martin et Henripin, m'avaient averti : fais attention tu vas te faire poser la question (à propos de la religion). C'est, qu'avant de crier sur les toits et de façon publique que tu étais agnostique ou 
athée ou marxiste ou autre chose, on te présumait catholique et on te laissait tranquille. Moi la liberté académique je l'ai toujours eue, je l'ai toujours exercée, je pense que j'étais probablement le seul dans le Département ou peut-être celui qui inspirait un peu de crainte, mais je n'ai jamais été brimé. Maintenant le fait de ne pas participer dans les grands débats publics s'explique, à mon avis, par la jeunesse; on avait 24-25 ans, on venait d'arriver des études et on enseignait 10-15 heures (par semaine). On essayait de faire un programme respectable... [...] Maintenant il y a eu le fait aussi que le nombre de professeurs changeait. Il y a des années où nous étions seulement nous deux, il y a eu d'autres années où on était cinq, d'autres années on était quatre avec quelques professeurs d'appoint et pas beaucoup d'étudiants. Les étudiants, eux, il n'y en avait pas beaucoup. Et évidemment on va toujours en sociologie un peu par indécision : on sait ce que l'on ne veut pas et on ne sait pas exactement ce que l'on veut. À l'époque les étudiants qu'on recrutait c'était ceux qui ne se sentaient pas satisfaits de faire service social ou relations industrielles! S'ils voulaient être un peu plus sécurisés ils allaient à relations industrielles ; le Père Bouvier avait monté une boîte qui avait l'air de mener à une carrière; ou ils allaient en service social qui commençait déjà à être professionnalisé. Dans la première vague d'étudiants, on retrouvait des personnes qui avaient un besoin de libération d'une certaine façon. La sociologie, pour les étudiants, ce n'est pas seulement la libération des peuples, c'est souvent la libération de l'individu: on voulait se sentir un peu libéré d'une certaine forme de catholicisme. Il y avait Mounier qui avait attaqué un peu le catholicisme traditionnel, Cité Libre, etc. et on était à l'intérieur de cet atmosphère-là.

M.R. - À la différence d'autres, j'avais affiché un certain agnosticisme déjà à mon retour d'Europe en 48 et un certain socialisme. J'avais été demandé à Laval, au Département de sociologie, par des collègues (Falardeau et les autres) et j'avais été refusé par le recteur du temps qui était un religieux. Ensuite à deux reprises on m'a demandé de venir à Montréal, dont une fois en 1956 par le Père Mailloux, pour devenir doyen de la faculté des sciences sociales et j'avais eu des entrevues avec Minville, avec Mgr Lussier, etc. Finalement, bien sûr, Lussier m'avait fait comprendre que le Conseil de l'Université, avant 61, était contrôlé de l'extérieur: duplessistes et intégristes. Je suis entré le 1er juin 1961 parce que l'abbé Lacoste, le cardinal Léger qui était chancelier (j'ai déjeûné avec lui) m’ont accepté. À ce moment-là certains journalistes, dont Louis Martin dans le McLean du temps, ont écrit que c'était la porte ouverte aux agnostiques, le premier agnostique reconnu qui l'avait crié sur les toits, à la radio ou à la télévision qui entrait à l'Université. On m'a bien honoré, je ne sais pas si c'est vrai, mais je vous rapporte ce que Martin écrivait.

R.S. - À partir de ce moment-là, nous entrons dans les années $60 \ldots$

M.R. - Cette période de la vie du Département coïncide avec un mouvement extraordinaire dans le Québec; c'est la Révolution tranquille qui 
commence, des gens d'Ottawa déménagent à Québec parce qu'il y a une Fonction publique qui va se bâtir, des enseignants qui avaient été refusés (dont Pierre Elliot Trudeau) avant 60 qui entrent à l'Université. Trudeau et moi avions fait un pacte: si on refuse l'un, l'autre ne vient pas. Comme nous avons été acceptés tous les deux, nous entrons à l'Université! La Révolution tranquille, ce n'est pas nous qui l'avons fait mais notre arrivée coïncide avec un déblocage de la société, une prise de la parole et un chambardement que je n'ai pas à décrire ici parce que tout le monde y a participé et a présent à l'esprit ce grand déblocage qu'a été l'arrivée au pouvoir de Lesage, de René Lévesque, de Paul Gérin-Lajoie par exemple. Évidemment la sociologie jusqu'à un certain point ou en tout cas l'Université de Montréal, peut-être à Laval, devient un endroit où on critique la société. Je me souviens qu'à Laval, il fallait, disaient les sociologues de l'époque, décrotter les étudiants de toute cette idéologie catholique qu'ils avaient absorbée dans les collèges classiques pour leur faire accepter une démarche inductive et critique de la société et il fallait aussi les préparer à embrayer avec la société et ceux, les hommes politiques ou les autres, qui étaient dans le mouvement, les syndicats par exemple, etc.

J.D. - C'est à peu près le moment où j'arrive et c'est évident que je n'ai pas manqué d'être frappé exactement par ce que Marcel vient de décrire, à savoir une société qui est en pleine ouverture, en pleine expansion; un milieu qui me frappe par sa tolérance, par des rapports non hiérarchiques, non autoritaires, moi qui venais de passer près de dix ans à Paris, en France, à faire de la recherche dans un milieu qui était certes ouvert mais dans une société qui ne l'était certainement pas comme on pouvait l'être ici. Et c'est pour moi très frappant : d'une part l'ouverture, deuxièmement la tolérance et troisièmement cette espèce d'enthousiasme qui régnait à ce moment-là. Tout était possible, tout vraiment semblait possible et tout était possible particulièrement en sociologie, en ce sens qu'on sentait bien le vent dans nos voiles et qu'on pouvait faire des projets, on pouvait aller chercher des fonds de recherches, on pouvait envisager l'expansion du Département. C'est une situation qui favorisait précisément des positions de grande tolérance parce que nous n'étions pas dans le Département des gens qui étaient en grande rivalité entre eux. L'expansion était là et, dans une période d'expansion, la rivalité est faible. Ça me frappait beaucoup. Une autre chose qui me frappait c'était le type de sociologie qu'on faisait par rapport à celle qui se pratiquait d'où je venais. Ce que je rencontrais, c'était de l'anthropologie sociale avec Marcel Rioux, c'était de la psychologie sociale avec Robert Sévigny, c'était Parson enseigné par Guy Rocher, c'était des relations ethniques chez Jacques Brazeau, toutes choses qui n'étaient pas très présentes finalement dans les milieux parisiens de sociologie à l'époque. En contrepartie je pourrais dire qu'il nous manquait de la sociologie économique, il manquait de l'histoire, il manquait de la science politique, il manquait une sociologie des classes sociales. On parlait tout à l'heure de l'introduction du marxisme, je me souviens que lorsque j'ai proposé de donner un cours de 
sociologie comparée des mouvements ouvriers et que j'avais indiqué comme lecture importante Karl Marx, le doyen Garigue m'avait appelé dans son bureau en me disant: "On n'a jamais enseigné Marx ici, ça ne passera jamais.» J'avais dit : «Essayez toujours on verra bien.» En réalité la Commission des études n'a pas fait d'objection finalement mais c'était symptomatique quand même que quelqu'un comme Garigue, qui était doyen puisse dire : "Ça ne s'est jamais enseigné.» Une autre chose m'avait frappé : le milieu étudiant; c'était un milieu assez bourgeois ou de petite bourgeoisie. J'ai gardé le souvenir de la première année de maîtrise, je donnais des cours où il y avait douze étudiants. C'était des étudiants qui avaient pratiquement tous été passer quelque temps dans un collège anglais de sœurs ou de prêtres bilingues. Ce n'est pas un hasard si, depuis quelques mois, il y a une contestation étudiante qui demande la suppression dans nos cours de tout texte en anglais. C'est qu'une partie de ces étudiants d'aujourd'hui ne vient pas des mêmes milieux sociaux que ceux d'autrefois. Ce que j'apportais était surtout de la sociologie du travail et une certaine sociologie des classes sociales venant d'un milieu où des gens comme Friedmann, Gurvitch, Lévi-Strauss, Aron étaient à leur apogée. Peut-être la chose la plus importante pour moi ce sont ces deux premières constatations, cette espèce de dynamisme incroyable qu'il y avait au Québec à ce moment-là qui vous donnait l'envie d'y rester, et c'est ce que j'ai fait, et cet autre horizon de théories sociologiques que je découvrais à travers mes collègues.

M.R. - Ce qui est frappant aussi, je crois que Jacques va être d'accord avec moi, et c'est peut-être le label de marque du Département de sociologie de l'Université de Montréal, c'est un pluralisme extrême, une très grande tolérance c'est-à-dire qu'on pouvait, dans une salle de cours, critiquer le fonctionnalisme d'un point de vue marxiste, ce que je faisais, et dans une autre salle l'abbé Lacoste faisant de la sociologie de la religion et Rocher faisant l'apologie de Parsons. Je crois que nous sommes arrivés à cette marque de tolérance et de civilisation me semble-t-il, d'avoir passé à travers ça et de nous être toujours très bien entendus, je dirais.

J.D. - C'est ça. Pour moi, cela a toujours été très important. On m'a plusieurs fois demandé, quand je rentrais en Europe, pourquoi je restais au Québec, et j'ai souvent répondu que je trouvais que c'était un milieu de travail exceptionnel par son ouverture et sa tolérance.

H.G. - Vous m'avez posé une question sur les années 50. Est-ce qu'il n'y a pas eu d'une façon un peu délibérée une rupture complète avec la sociologie américaine; c'est l'image qui s'est dégagée d'autres milieux sociologiques. À peu près au moment où il y a eu des fissures au niveau des associations volontaires comme la Chambre de commerce canadienne, il y a eu un «tournant» autour de 63-64 ou 65. Après ça il n'y avait presque plus d'étudiants gradués qui allaient vers les universités américaines. La plupart allait découvrir la sociologie américaine en Europe. 
M.F. - Je disais que la sociologie américaine a toujours été représentée chez nous par Rocher, par Brazeau, par Mlle Massé entre autres. Mais que nos étudiants soient allés plutôt se former en Europe, je crois que c'est exact aussi, bien que quelqu'un comme Carlos, un de nos meilleurs étudiants, soit allé à Chicago. Mais il n'y a pas rupture totale et je crois que, à l'heure actuelle, il y a plutôt une espèce d'équilibre; la sociologie américaine a repris du poil de la bête à travers Paul Bernard, Marc Renaud et d'autres.

J.D - Peut-être aussi, une chose qu'on pourrait mentionner, c'est que après toutes ces années, 60 c'est l'année où l'on voit émerger sur la scène sociologique internationale, une jeune sociologie française qui attire beaucoup de gens, comme Crozier, Moscovici, Barthes, Morin, Touraine. C'est une jeune sociologie qui se fait en français et comme au fil des années l'attrait vers la langue française marque le Québec très fortement, l'attrait vers cette jeune sociologie française se renforce.

R.S. - Il faudrait peut-être dire que le problème de la langue s'est posé au Département il y a longtemps, et que dans les années 60 , à peu près tous les noms de sociologues français que tu as mentionnés sont venus ici comme professeurs invités pendant une certaine période alors qu'il n'y avait pas d'équivalents chez les Américains.

M.R. - Parce que les Américains ne sont pas naturellement bilingues.

J.D. - Il n'y a pas seulement ça. Il y a une politique du gouvernement québécois qui facilite au maximum la venue de professeurs français. Il y a des accords France-Québec qui sont mis en place et qui donnent la possibilité de faire venir des professeurs; on n'a pas l'occasion de le faire avec des Américains, à si bon compte.

N.L. - Il y a un aspect dont il faudrait parler à l'occasion de la Révolution tranquille: la contribution des sociologues du Département sur la place publique. Entre autre, je pense à la Commission Parent. Rocher avait un grand prestige; il était un excellent professeur; à ses cours d'introduction, c'était salle comble et il avait aussi une grande réputation à cause de son rôle important dans la Commission Parent. Les sociologues étaient les nouveaux sorciers du village. Ils étaient conscrits pour des travaux, et moi-même $\mathbf{j}$ 'ai fait des travaux pour la Commission Parent, sur la restructuration scolaire de l'île de Montréal. Marcel Rioux, pour sa part, avait dirigé une Commission sur l'enseignement des Arts. Tout cela donnait aux sociologues presque une parole d'évangile.

J.D. - Sinon une auréole. Dans le même sens on pourrait dire que c'est le moment où la télévision utilise le plus les sociologues. Nous sommes certainement presque tous, dans cette période-là, passés quoi, 4 ou 5 fois au moins, durant l'année dans des émissions diverses. Nous étions très très souvent sollicités, beaucoup plus que nous l'avons été, postérieurement je crois, et ça mettait la sociologie sur la place publique.

M.R. - Parce que dans le fond, 60-68 c'est l'âge des sociologues et des idéologues; parfois dans le public on pensait que sociologie égalait socialis- 
me. Par rapport à nous, par rapport à l'Université de Montréal et on avait une action publique que la force des choses nous donnait tant par notre action dans la cité que, comme dit Jacques, par nos apparitions fréquentes à la télévision et à la radio.

J.D. - C'est le moment où l'on forme un club socialiste à l'Université qui s'appelait «Le club Jacques Perrault». C'est quand même une chose nouvelle.

M.R. - Et nous participons, Jacques et moi, au N.P.D. On fonde le P.S.Q., on fonde une revue Socialisme international et québécois et on est impliqué beaucoup dans la cité. On collabore à Parti pris; vous voyez que c'est toute cette époque qu'il faut ressusciter.

R.S. - En même temps pour moi 68 rappelle les mouvements étudiants. Les gens se réfèrent souvent à mai 68 en parlant de la France et de Berkeley. Il faudrait se souvenir qu'au Département de sociologie, je ne sais pas si c'est un honneur, mais au Département de sociologie, 68 ç'a été d'abord février 68 où il y a eu une première très grosse et très dure contestation étudiante qui a été ensuite suivie de mai 68 en France, et qui a repris ici en octobre 68 . Alors sur ce thème des mouvements étudiants, on y a fait allusion tantôt en parlant déjà des années 50-55, comment voyez-vous ça maintenant?

N.L. - Pour ma part, j'ai une anecdote. Je me rappelle avoir cherché un assistant de recherche pour un de mes cours sur l'histoire et l'organisation de la société québécoise. Je recrute un jeune anglophone qui parlait très bien le français et qui avait une formation économique et qui me semblait fort brillant. J'arrive au premier cours; il arborait avec fierté la faucille et le marteau alors que moi j'étais en collet romain. Craignant avoir des conflits sur l'interprétation de la société québécoise on a fait un pacte : je lui ai dit: «Es-tu capable de donner une bonne lecture marxiste du Québec?»Il a dit oui. "Quant à moi je vais donner une bonne lecture wébérienne.» Cela a été un des cours des plus intéressants qu'il y a eus car ç'a été vraiment une double lecture de la société québécoise. C'est le genre de réalisation possible je pense, presque uniquement chez nous.

H.G. - Durant les années 50, je fais référence aux années où j'étais ici, le problème c'était la redéfinition du catholicisme ou d'en sortir. C'était le grand problème idéologique de la jeunesse comme vous disiez. Ils sortaient des collèges classiques et, en sciences sociales du moins et particulièrement en sociologie, se posaient tous le problème de redéfinir le catholicisme ou d'en sortir. C'était une des tensions qu'on sentait chez les étudiants. Il y avait, disons, des agnostiques cachés, quelques militants mais presque pas, il y avait des catholiques de gauche, il y avait également des mouvements de droite. Il y avait eu à un moment donné une peur de la montée de l'intégrisme; là on entre dans les conflits qui étaient abordés dans Cité libre. Il y avait Pax Romana qui avait été puissante chez les étudiants. Je me souviens d'un type, je ne veux pas vous le nommer, de toute façon je ne me souviens plus 
de son nom mais qui en avait été membre. Il m'a annoncé plus tard qu'on avait discuté de mes cours à une assemblée parce qu'on ne me trouvait pas très catholique ou trop «catholique» et on avait décidé de ne rien faire. Lui était à ce moment-là du côté assez intégriste de ces associations étudiantes-là. Alors je l'ai rencontré à Paris, il était devenu agnostique, athée et psychanalyste, etc. Ce qui veut dire qu'il y a eu au niveau des étudiants, sans que cela ne prenne peut-être une forme de contestation publique, beaucoup de tiraillements individuels qui étaient assez généralisés pour qu'on le sente, mais sans que ce soit jamais une chose qui éclate. Je dois dire au niveau de l'institution que tous ces conflits étaient peut-être latents et sous-jacents mais qu'il n'y en avait pas... C'est la venue de ce fameux Hudon-là, qui était plus connu que le recteur, qui les a rendu visibles. Il est devenu une «institution» en l'espace d'un an, et il disait qu'on était une université catholique, qu'il voulait proclamer ça. Il voulait arrêter les clubs de la rue Saint-Laurent où il commençait à y avoir des danseuses à go go. Il avait un groupe $d$ ' «hommes-forts» qui se promenaient dans la ville pour protéger la moralité. Vous souvenez-vous d'Hudon? C'était un gentil garçon, il était en économique d'ailleurs et n'était pas en sociologie.

R.S. - Je pense en vous écoutant au film Seul ou avec d'autres qui a été fait juste à la fin de cette période-là et que j'ai eu l'occasion de revoir l'an dernier; il illustre bien, je pense, le climat que tu décris.

J.D. - Il y a un autre point et cela enchaîne sur ce que l'on vient de dire, c'est que si on replace les étudiants d'aujourd'hui dans les années 60 , la grande différence c'est l'existence d'un mouvement, d'une organisation étudiante interne et très active dans l'Université, c'est l'existence d'un journal qui s'appelle le Quartier latin, qui est l'affaire des étudiants, qui, pendant un certain temps, a comme rédacteur en chef notre collègue Paul Bernard et où l'on retrouvait, si on regardait les comités de rédaction, à peu près une bonne partie du personnel du gouvernement actuel, des Landry, Marois et Cie, tous ces gens qui sont sortis et passés par ces associations étudiantes. Elles ont disparu après 68 et ne sont pas encore vraiment revenues sur la carte.

M.R. - 68, je crois que c'est là-dessus que vous nous interrogiez. Bon je crois que c'est, bien sûr, février 68, à Montréal et, mai 68, à Paris. Je me souviens d'avoir fait un triplex à la radio avec Touraine à Paris et quelqu'un d'autre à New York sur ces mouvements-là; étant québécois, je leur rappelais que cela avait commencé ici avant mai 68. Mais plus généralement ce sont les conflits de génération qui commencent, et la contestation de génération ça va jusque dans le clergé. Je me souviens par exemple d'avoir été invité par les jeunes prêtres de Montréal à leur parler de la société et j'avais le vicaire de l'abbé Lacoste qui le contestait déjà, et j'ai dû prendre parti pour l'abbé Lacoste parce qu'il était mon collègue, etc. Une autre fois je suis invité par 95 supérieurs de couvents et de collège à Chateauguay dans une grande maison pour leur parler de la jeunesse et de l'avenir du Québec. Comme mon nom 
était venu souvent pour traiter de ces deux problèmes-là, les religieux disaient : «Mais qu'est-ce qu'on fait, c'est un agnostique, c'est un socialiste, c'est un indépendantiste." Alors ils étaient allés voir un dominicain. Le dominicain leur dit: «C'est un homme poli qui vous dira ce qu'il croit savoir mais ce n'est pas l'homme à tirer des hosties et des ciboires. » J'étais allé là et, je pense, que c'est peut-être le seul endroit au monde où je pouvais parler en marxiste à des supérieurs de couvents et de collèges classiques sans qu'il y ait la moindre contestation tellement l'ouverture d'esprit était grande. Mais je reviens sur mes pieds! Il y a deux ou trois ans on m'a demandé d'écrire un article pour Parti pris avec Paul Chamberland sur l'aventure de Parti pris. Mon verdict avait été que les collaborateurs et le Conseil de direction n'avaient pas analysé leur écriture mais étaient eux-mêmes devenus leur écriture, se privant de l'analyse. De sorte que au lieu de faire une revue, Chamberland, par exemple, s'est habillé d'une toge et est parti dans le maquis. Pour moi c'est un moment extrêmement important du côté de la sociologie, parce qu'on se souvient des articles de Nanterre «Pourquoi les sociologues», on se souvient que la sociologie est mise en cause à Paris, elle est mise en cause ici et il y a ce conflit de génération qui aboutit, qui avait commencé en 64 à Berkeley. Pour moi c'est un moment très important et au niveau social et au niveau de l'enseignement et de la pratique de la sociologie.

H. G. - Est-ce qu'il ne faudrait pas ajouter à ça le FLQ? Parce que l'incident du FLQ...

M.R. - Mais c'était commencé en 64 avec l'Armée de libération du Québec...

H.G. - Tout ce que je veux dire c'est qu'il y a le mouvement étudiant, mais à propos des relations entre sociologues et société, l'intervention du FLQ a eu des effets. Après le FLQ, cela n'a jamais été pareil. Il y a une retombée beaucoup plus technicienne, professionnelle qui s'accentue d'ailleurs.

M.R. - Moi je diffère un peu d'opinion avec Hubert. C'est que dans le court temps, c'est bien sûr que l'arrivée de l'armée ici et les événements d'octobre ont fait peur à la population et cela s'est calmé. On le voit par exemple au niveau politique, 103 ou 102 députés sur 110 au Parti libéral en 1973. Trois ans plus tard la société est revenue de sa peur et toutes sortes de messages apparaissent dans la société. Moi j’ai un faible pour les artistes et particulièrement pour le film de Michel Brault qui réalise les Ordres. C'est un film qui est un flop absolu à Paris quoique piloté par Lelouch, je crois, mais ici c'est un film qui parle aux gens parce qu'il s'agit de la vie familiale, il s'agit des individus. Ça ne rappelle pas de grands événements, mais je crois que tout ça est accumulé dans la conscience collective. Il semble que dans un premier temps on fait peur au monde et les gens ont peur, et après on est pas mal paysan et têtu et on continue. Et en 1976, ce gouvernement qui semblait là pour 25 ans s'écroule complètement. 
R.S. - Depuis un certain temps, vous brossez un tableau au fond de l'arrrière scène sociale et politique du Québec. La question que je me pose maintenant c'est : le Département de sociologie ou un Département de sociologie à travers ces périodes-là, ça devient quoi? Vous mentionnez ces événements-là parce que vous voyiez le Département comme étant un peu déterminé par ces événements-là ou au contraire comme étant...

N.L. - Une réalité est évidente c'est la grande différence entre la documentation des années 60 et celle des années 80 . Je me rappelle avoir donné un cours en 1960, et devoir chercher des sources étrangères sur le Québec et ne trouver que l'une ou l'autre monographie des étudiants. Aujourd'hui on a une documentation de premier ordre, accessible aux étudiants; cela donne une sécurité beaucoup plus grande, une organisation du matériel beaucoup plus cohérente, enfin les explications de la société sont maintenant bien faites. À ce moment-là, le professeur lançait ses hypothèses à partir de l'une ou l'autre théorie qu'il connaissait tandis que maintenant c'est bien identifié, et bien expliqué, c'est vraiment très différent.

H.G. - Moi c'est peut-être une autre perspective, une perspective de l'extérieur. Par nécessité, je fréquente beaucoup plus le milieu sociologique anglophone que francophone. Et ce qui se dégage c'est une image d'envie de la sociologie québécoise, une image qui est à la fois une sociologie cohérente mais surtout qui est engagée par rapport à sa société. Eux, déplorent que ça n'existe pas chez eux. Cela couvre la gamme idéologique des sociologues anglophones. Tout le monde, que ce soit ceux de droite - il n'y a pas de sociologues qui se définissent de la droite mais ils le sont des fois -, du centre ou de la gauche, il y a une envie. Moi j'ai toujours pensé que, peut-être, la différence vient de la question nationale, de la question de minorité, de la question de la société, peut-être aussi de la question des origines sociales de la sociologie d'ailleurs. C'est la sociologie du reste du Canada qui n'est pas vraiment une sociologie canadienne, même pas au niveau de ceux qui l'enseignent. La proportion de ceux qui viennent des États-Unis et d'ailleurs et qui font l'enseignement de la sociologie, peut-être débalance cette relation de la sociologie et de la société et je ne sais pas ce que vous en pensez; je pense que cette relation a été exprimée beaucoup par l'action du Département de sociologie, beaucoup plus de Montréal que de Laval disons de 62-63 jusqu'à 70 et même après ça.

M. R. - Il y a aussi l'autre fait. Moi j'ai enseigné à Carleton, et je me suis rendu compte que les Américains, comme à Calgary, étaient dans les Départements de sociologie extrêmement nombreux. La blague était qu'on avait engagé à Calgary le $31^{\mathrm{e}}$ sociologue et que celui-là était Canadien ! Et cela n'a pas beaucoup changé parce que, l'an dernier, je suis allé à Calgary et les Canadiens me prenaient à part et me disaient qu'ils étaient toujours dominés par eux. Mais il demeure qu'il y a beaucoup d'Américains examinant la société canadienne en pièces détachées, disons les syndicats canadiens par rapport aux syndicats américains, le système d'éducation canadien par rapport à l'américain, etc. Ils exami- 
nent la société en pièces détachées. Tandis que, nous ici, notre approche est beaucoup plus globaliste. Nous n'avons peut être pas de mérite parce que le nous, il est déjà dans la société, le nous québécois, on ne peut pas passer à côté, de sorte que par cet aspect global, on regarde chaque pièce détachée par rapport au tout et c'est ça qui fait la différence, et fait l'envie en tout cas de Vallée et Whyte (les anciens directeurs du Département de Carleton) et de bien d'autres sociologues. Je vais vous donner un exemple tout à fait récent. Après 76 , je m'en vais à Toronto, j'essaie de conscrire Rostein de la Massey Foundation qui est un économiste, qui est nationaliste canadien pour l'entraîner à notre suite (je poursuis toujours le même rêve), mais ce sont des gens qui restent dans leur bureau. Rostein et d'autres me disent : "Qu'est-ce qu'il faut faire? »Sortez de vos sacrées universités et cessez de vous convaincre entre vous. Essayez d'aller à la télévision et à la radio, de noyauter certains groupes; ce qu'ils ne semblent pas pouvoir faire, même encore aujourd'hui. Je parle beaucoup avec des gens de ces problèmes; j'en ai rencontré deux, l'autre jour, qui venaient de York et de Sudbury University. Ce sont des gens qui me semblent tout à fait renfermés dans leur tour d'ivoire par rapport à ce que nous ici du Québec avons toujours fait. Il y a toujours de la communication de sorte que cet influencelà je suis bien heureux que ce soit Hubert de l'extérieur qui la mentionne, c'est un peu notre label de marque.

R.S. - À ça Marcel, j'ajouterais que, même dans le cas où il semble que des collègues du Département qui ont des champs plus spécialisés, santé, travail, éducation, psychologie sociale, même dans ces cas-là, il y a une forme de contact avec les milieux. Même si chacun n'a pas cette approche aussi globalisante que celle que vous mentionniez, mais même dans le cas des autres, il y a cette forme d'ancrage avec un milieu.

H.G. - Je pense qu'une des différences c'est que le contact n'est pas seulement à travers l'instrument de recherche. Tandis que chez les sociologues en Ontario j'ai l'impression que c'est beaucoup l'instrument de recherche qui crée le contact avec le milieu.

N.L. - Dans le domaine de la sociologie de la religion les formes d'engagement pastoral que j'ai eues et mes responsabilités m'ont été une source d'enrichissement très grand. Quand on nous voit venir comme un sociologue patenté, on n'a pas la même réaction que si on est simplement le participant d'une action.

J.D. - La chose que je voulais dire aussi sur ces années 60 et un peu après c'est la variété des influences théoriques qui sont exercées sur la discipline et sur l'enseignement de la sociologie. Rappelez-vous le début des années 60 , sans doute que les théoriciens dont on parle seraient plutôt soit C.W. Mills, Parsons, E. Hughes et de l'autre côté des gens comme Gurvitch, Lefebvre, Aron, mais ensuite il y a, je crois, ceux dont j'ai parlé tout à l'heure, la génération qui suit. Mais après 68, il y a une rupture et il y a apparition du néo-marxisme, et il y a ce grand courant althusérien et poulantzacien qui balaie le Québec mais 
qui balaie plus particulièrement l'UQAM. L'UQAM est très poulantzacienne; ça correspond à la suite des événements de 68 , mais ça correspond aussi, je crois, à des événements politiques importants, une espèce de renouveau socialiste en Europe. C'est une période où il y a beaucoup d'espoir qui se traduit aussi au plan théorique. Et puis ce courant-là se tarit, c'est la dégringolade du stalinisme, du léninisme, et même d'un certain marxisme, Poulantzas notamment. Ensuite trois influences se font sentir: il y a d'une part un renouveau de l'apport de la sociologie américaine par son rapprochement avec la biologie, d'un autre côté il y a l'apparition d'un marxisme américain qui se diffuse et qui nous semble à nous parfois naïf mais vif, alerte, jeune, et troisièmement il y a quelque chose de complètement différent qui apparaît sur la carte et est en train de s'étendre, c'est une réflexion, c'est un marxisme militant, c'est le courant maoïste et trotskyste qui se répand dans les collèges, les Cegeps, etc. qui nous apporte dans les salles de cours des gens qui attendent quelque chose à ce sujet-là.

R.S. - Qui ne nous a pas encore fourni de professeurs.

J.D. - Non qui ne nous a pas encore apporté de professeurs effectivement, mais il y a une espèce de dégradation dans la qualité de ce marxisme, car c'est un marxisme militant qui est très pauvre intellectuellement et peut être aujourd'hui on est dans un «no man's land» théorique. Je ne sais pas si je me trompe mais j'ai un peu l'impression en tout cas que le champ est plus ouvert qu'il ne l'a jamais été; il n'y a plus aucune espèce d'influence qui me paraîtrait déterminante comme on aurait pu le dire en d'autres temps. Il y a sûrement une période où Althusser a été un des champions de la pensée marxiste occidentale. Aujourd'hui c'est plutôt un vide, un vide qui répond à la crise de la sociologie... comme si la sociologie était un peu arrivée au bout de son discours... certains le disent. On va chercher de plus en plus ailleurs des choses à comprendre, physique, biologie, histoire, des choses à expliquer avec un bagage, certes sociologique, mais qui n'est peut-être plus le discours purement sociologique très caractéristique des années 60-80.

R.S. - Là tu parles à la fois de la sociologie en général et de ce qui se passe ici?

J.D. - Ici au Québec, et ailleurs.

N.L. - Pour ma part, j'ai abandonné pendant quelques années l'enseignement de la sociologie de la religion. Rocher m'avait dit: "Ça n'intéresse plus personne» et effectivement à une époque, c'était vrai. Mais devant les mouvements plus récents je me suis dit : «Il est important de refaire une analyse sociologique du mouvement spiritualiste qui est en train de naître» et je dois dire que les étudiants manifestent un intérêt de ce côté-là. On a beaucoup étudié la réalité matérielle avec l'aide de la sociologie, j'ai l'impression que, maintenant, il faut faire une étude de tout ce renouveau spirituel et je dois dire que la sociologie traditionnelle n'était pas très très bien équipée de ce côté-là. L'anthropologie 
pourrait peut-être fournir des modèles intéressants. Je pense à toutes les visions du monde, au néoidéalisme... Je suis très optimiste sur la contribution future de la sociologie. Il y a cependant une condition : celle de se renouveler dans son objet et dans sa méthodologie.

R.S. - Marcel, vous voulez réagir à ce qui vient d'être dit?

M.R. - J'enchaîne sur ce que Jacques dit. C'est qu'on a assisté à la désaffection, si on peut dire, d'un maître-penseur, c'est-à-dire d'une certaine conception, je parle du courant marxiste, d'une certaine conception de la détermination des faits sociaux. On s'est rendu compte que le social-historique n'est pas «théorisable» de part en part et la pratique redevient importante par rapport à la théorie; si vous étiez un marxiste orthodoxe, Marx avait décidé quel était le sujet historique, comment ça devait se passer et tout ce qui s'en suit. On a un peu déchanté de tout cela et, à l'heure actuelle, dans ce que j'enseigne et qui m'intéresse, disons la sociologie critique, je m'intéresse davantage aux groupes et aux conduites «émancipatoires", c'est-à-dire qui sont définis soit par les idéaux de la société ou par des jugements de valeur. Dans ce courant (la sociologie critique) on ne fait pas faute de lier fait, valeur et intervention. Si je prends par exemple ce qui se passe à Paris, je vois qu'il y a beaucoup de gens qui définissent leur groupe et la société comme une société d'expérimentation; non seulement il y a désaffection envers les maîtres-penseurs du passé et du présent mais aussi envers les appareils politiques (c'est pour ça que le parti socialiste et $M$. Mitterand et Marchais et tout ce beau monde-là sont en état de désaffection); les gens cherchent plutôt au ras des pratiques quotidiennes, innovatrices, etc. ce qui apparaît de nouveau et d'émancipatoire. À mon avis, et c'est ce que je fais, c'est un peu un réalignement de la sociologie vers les pratiques dites émancipatoires : c'est le courant de l'École de Francfort sans le désenchantement ni de Weber, ni d'Adorno, ni même de Marcuse. Ce courant s'oriente plutôt vers la recherche de sujets historiques éclatés; le prolétariat, les jeunes, les chicanos, les femmes, etc. Touraine, par exemple, ne fait plus de grands traités et cela me paraît extrêmement révélateur de ces nouvelles directions de la sociologie.

H.G. - Un peu dans la même ligne mais plutôt sous forme de question. Il y a eu quand même les nouveaux philosophes et la position anti-étatique qui est très forte chez les économistes. À mon avis, c'est comme un contre-courant que j'ai remarqué dans mon institution. De 60 à 73, j'avais une gauche articulée parmi les étudiants et une droite inarticulée. Depuis 2 ou 3 ans ce qui me frappe, les étudiants qui sont articulés sont dans le courant de droite et il y a des étudiants qui sont émotivement de gauche mais inarticulés intellectuellement.

R.S. - Je n'ai pas l'impression de retrouver, à l'heure actuelle, à l'intérieur du Département, la pensée des nouveaux philosophes.

M.R. - Moi j'irais plus loin que ça, je dirais que, par rapport aux années passées, nos étudiants ne sont pas aussi articulés, ni ceux de gauche, ni ceux de droite. 
R.S. - J'aurais le goût de vous proposer un dernier tour de table pour répondre à cette question: à part les réflexions que vous avez déjà faites sur le Département, est-ce qu'il y a des éléments critiques que vous souhaiteriez lancer sur la table?

J.D. - Moi j'ai une réflexion qui porterait sur les étudiants. J'ai l'impression que toute la population étudiante du Département de sociologie a toujours été divisée, non pas en terme de conflit mais en terme de ceux qui cherchaient une formation théorique assez forte, une méthode spéculative, une méthode d'observation et d'autres qui cherchaient une voie professionnelle. Et il semble qu'aujourd'hui les choses arrivent à un point presque de tension et de contradiction incroyable entre d'une part des étudiants qui voudraient avoir une sociologie très proche de la pratique de l'action, etc. et en même temps des étudiants qui aspirent en sortant de leurs études à avoir une profession reconnue, un emploi. La pression de la vie économique, du chômage, de l'inflation et aussi du surplus des sociologues est telle qu'il y a une ambiguité profonde dans l'attitude étudiante: d'un côté une critique vigoureuse, agressive de la société, des conditions qui leur sont faites et, en même temps et paradoxalement, l'exigence qu'on leur donne quelque chose qui va leur assurer un emploi. C'est une des choses qui me frappe dans la population étudiante d'aujourd'hui par rapport à la population étudiante d'il y a 20 ans, privilégiée, peu nombreuse. C'était une sociologie ouverte, c'était la conquête d'un champ scientifique.

H. G. - Ce que je verrais et ce qui me préoccupe actuellement c'est le thème de la société traditionnelle. Je sens de plus en plus le besoin de mettre en cause l'interprétation que l'on a faite dans les années 50 ou au début des années 60 de la société traditionnelle. Et j'ai l'impression que, dans le feu de l'action, le marxisme n'a pas fait mieux non plus. Au fond, l'histoire de la société québécoise, disons des cent dernières années, est complètement à refaire. On peut voir, avec 30 ans ou 20 ans de recul, que les sciences sociales et particulièrement la sociologie ont dressé le tableau de cette société, de ses principaux acteurs et de ses institutions de base pour s'en libérer politiquement. Mais ceci ayant été fait, je pense qu'il y a un besoin de retrouver l'histoire. Je pense, que, dans un $2^{\mathrm{e}}$ temps - et cela sera fait probablement par nos successeurs - il y aura un certain équilibre. D'ailleurs il y a une tradition en histoire présentement où il se fait une meilleure histoire et $\mathrm{j}$ 'ai l'impression que l'histoire aura pour fonction d'équilibrer la médiation entre la sociologie et la société.

M.R. - Moi je voudrais terminer brièvement sur les considérations peut être plus théoriques, plus historiques. La sociologie est née après les mutations sociales qu'ont apportées la révolution industrielle et la révolution française (pour rester en Europe). Prenons ceux que Gurvitch appelait les grands fondateurs : St-Simon, Proudhon, Marx, Durkheim et Weber. Ils vivaient post-factum et ils analysaient les bouleversements qui étaient en train de se produire et qui s'étaient déjà produits depuis la Révolution française. La sociologie d'alors est devenue tradition- 
nelle, autant celle des fonctionnalistes que celle des marxistes; elle est mal placée pour étudier les mutations culturelles et autres dans la société d'aujourd'hui, parce que cela ne s'est jamais encore produit. Cette sociologie est mal placée, pour parler comme Gramsci, pour voir ou pour essayer de détecter ce qui meurt et ce qui veut naître, et c'est pour cela que les maitres-penseurs sont disparus. Si tant est que nous soyons dans une mutation culturelle, un mode de production qui est allé au bout de son champ, alors il faut de nécessité utiliser une autre approche sociologique. Peut-être que cette approche ne s'appelle pas «sociologique» mais essaye de percevoir ce qui veut naître et d'épauler ce qui veut naître. Les sociologies traditionnelles sont mal placées pour ce faire. Et je voudrais réagir justement à ce que disait Hubert: je crois qu'il a raison et je crois aussi qu'il est en accord avec Fernand Dumont. À ce moment-là, moi aussi j'ai réagi, j'ai analysé la société traditionnelle et j'en ai étudié des formes; mais je me sens plus prêt que je l'étais à faire une autre analyse. Mais la différence entre moi et certains collègues, c'est que je ne veux pas retourner en arrière. Et ce qui me fait peur, à cet égard, c'est la religion. Si par exemple des sociologues ou d'autres personnes disent : "Voilà, ce qui a fait la grandeur du Québec c'est la religion - et je ne dis pas ça pour vous Hubert mais, peut-être, pour l'abbé Lacoste et ceux qui exagèrent davantage dans ce sens-là parce que l'abbé Lacoste n'exagère peut-être pas trop - c'est que ça veut dire qu'on retourne à la religion, que les femmes se remettent à avoir des enfants et on repart vers le Moyen Âge! Je caricature mais je crois qu'il y a une réflexion à faire sur cette société québécoise qui n'est pas aussi érodée, aussi défaite que la société américaine, sur ce qu'elle a encore de traditionnel et sur lequel on peut bâtir. Si on regarde la société américaine et si on regarde ce que le Québec est et ce qu'il a conservé des anciennes valeurs et des anciens modes d'être en société, on est en avance. Alors moi je me dis : «Pourquoi aller jusqu'au bout des Américains qui cherchent déjà dans notre sens pour revenir les imiter ensuite? $\gg$ On se fait alors des vocations de «rattrappeux» jusqu'à la fin du monde! Pour moi, l'objectif c'est le dépassement et le moyen c'est la traverse qui est un chemin plus direct vers un but, au lieu de la route américaine principale.

R.S. - C'est un projet pour la sociologie du futur.

M. R. - On a commencé à la faire.

N.L. - Marcel met en cause la religion. Je pense qu'il ne tient pas suffisamment compte des changements profonds qu'il y a eu dans la religion depuis 20 ans, et ça c'est très important et c'est une des raisons pour lesquelles j'ai tenu à ce qu'il y ait un cours de sociologie. de la religion; c'est qu'il y a eu vraiment une mutation au sein de la pensée religieuse. Les dogmes essentiels sont les mêmes mais les formes ont évolué. Je pense au renouveau biblique qui a complètement renouvelé l'ancienne perspective par une vision beaucoup plus anthropologique à partir de toute la tradition biblique. Je suis convaincu que si on veut redonner une lecture à cette société qui est en train de naitre il va 
falloir réévaluer l'ancienne religion que l'on a critiquée, dans certain cas, avec raison. Je pense qu'il faut faire les nouvelles lectures apportées par les théologiens des différents pays et qui peuvent nous permettre d'assimiler des valeurs qui nous étaient traditionnelles mais dans un mode plus libérateur que celui qu'on a connu.

M. R. - Vous me permettrez, Monsieur le meneur de jeu, de signifier mon désaccord avec les idées que vient d'exprimer monsieur l'abbé Lacoste.

R.S. - Nous sommes arrivés spontanément au dernier thème que je souhaitais aborder, celui du développement de la sociologie. Il est assez significatif que nous ayons terminé cette discussion en abordant autant le futur de la société que le futur de la sociologie. Je vous remercie.

\section{RÉSUMÉ}

Publié à l'occasion du vingt-cinquième anniversaire du Département de sociologie de l'Université de Montréal, ce numéro porte sur l'histoire et le développement de ce Département. Ce document prend la forme d'une table ronde à laquelle ont participé Jacques Dofny, Hubert Guindon, Norbert Lacoste et Marcel Rioux. On y trouvera des références aux divers courants sociologiques qui influencèrent (ou non) les premières années de ce Département, aux positions prises par divers acteurs de l'institution universitaire, au contexte social et culturel du Québec de la fin des années cinquante, aux mouvements étudiants, etc. Ce document présente à la fois des éléments d'analyse et des témoignages personnels de personnes qui ont toutes été intimememt liées au Département de sociologie dans les premières années de son existence. Cette table ronde était animée par Robert Sévigny.

\section{SUMMARY}

In this issue published on the occasion of the twenty-fifth anniversary of the Department of Sociology of the University of Montreal, we thought it would be interesting to include a document on the history and development of the department. This document has taken the form of a round table in which Jacques Dofny, Hubert Guindon, Norbert Lacoste and Marcel Rioux participated. Reference is made to the various sociological trends that influenced (or did not influence) the first years of the department, to the positions taken by the various actors within the university, to the social and cultural context in Quebec at the end of the 1950 's, to the student movements, etc. The document presents both analytical elements and personal accounts of individuals who were clos»ly connected to the Department of Sociology in the first years of its existence. The round table was animated by Robert Sévigny.

\section{RESUMEN}

En este número publicado con ocasión del veinticinco-avo aniversario del Departamento de Sociología de la Universidad de Montreal, hemos pensado incluir un documento sobre su historia y su desarrollo. Este documento toma la forma de una mesa redonda en la cual han participado Jacques Dofny, Hubert Guindon, Norbert Lacoste y Marcel Rioux. En el se encontrarán referencias sobre: las diversas corrientes sociológicas que han influenciado (o no) los primeros años del Departamento; las posiciones tomadas por diversos actores de la institución universitaria; el contexto social y cultural de Quebec a fines de los años cincuenta: los movimientos estudiantiles etc. Este documento presenta al mismo tiempo, elementos de análisis y testimonios personales de aquellos que estuvieron íntimamente ligados al Departamento de Sociología en los primeros años de su existencia. Esta mesa redonda fue dirigida por Robert Sévigny. 